\title{
A Model for the Shapes of Advected Triangles
}

\section{Alain Pumir \& Michael Wilkinson}

Journal of Statistical Physics

1

ISSN 0022-4715

J Stat Phys

DOI 10.1007/s10955-013-0789-6

Volume 152 - Number 2 - July 20

ONLINE FIRST

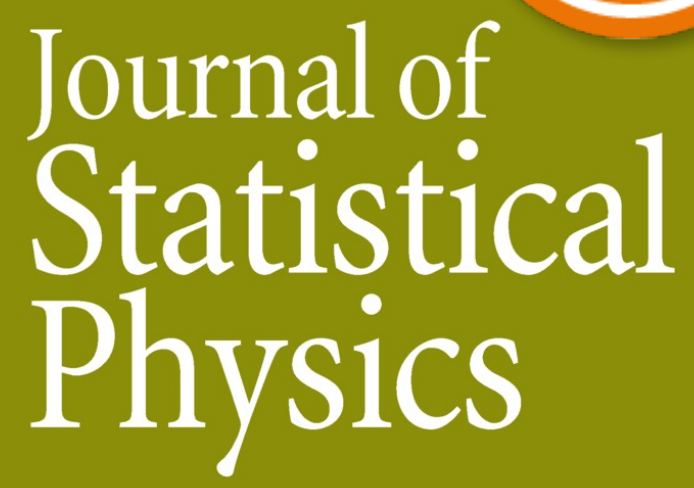

Journal of Statistical Physics 
Your article is protected by copyright and all rights are held exclusively by Springer Science +Business Media New York. This e-offprint is for personal use only and shall not be selfarchived in electronic repositories. If you wish to self-archive your article, please use the accepted manuscript version for posting on your own website. You may further deposit the accepted manuscript version in any repository, provided it is only made publicly available 12 months after official publication or later and provided acknowledgement is given to the original source of publication and a link is inserted to the published article on Springer's website. The link must be accompanied by the following text: "The final publication is available at link.springer.com". 


\title{
A Model for the Shapes of Advected Triangles
}

\author{
Alain Pumir • Michael Wilkinson
}

Received: 2 March 2013 / Accepted: 17 June 2013

(C) Springer Science+Business Media New York 2013

\begin{abstract}
Three particles floating on a fluid surface define a triangle. The aim of this paper is to characterise the shape of the triangle, defined by two of its angles, as the three vertices are subject to a complex or turbulent motion. We consider a simple class of models for this process, involving a combination of a random strain of the fluid and Brownian motion of the particles. Following D.G. Kendall, we map the space of triangles to a sphere, whose equator corresponds to degenerate triangles with colinear vertices, with equilaterals at the poles. We map our model to a diffusion process on the surface of the sphere and find an exact solution for the shape distribution. Whereas the action of the random strain tends to make the shape of the triangles infinitely elongated, in the presence of a Brownian diffusion of the vertices, the model has an equilibrium distribution of shapes. We determine here exactly this shape distribution in the simple case where the increments of the strain are diffusive.
\end{abstract}

Keywords Diffusion · Advection

\section{Introduction}

Consider motion of three tracer particles floating on the surface of a fluid which exhibits a complex flow [1-3]. The three points form a triangle whose shape is specified by two angles, and is therefore specified by a point in a two-dimensional manifold. It is of interest to consider the evolution of the shape of this triangle, independent of its scale, rotation and translation of its origin, because this is a means to characterise the essential geometry of the flow. Early investigations of random shapes were mathematical [4-6], and originally motivated by discerning whether nearly co-linear arrangements of archaeological features

\footnotetext{
A. Pumir $(\varangle)$

Laboratoire de Physique, Ecole Normale Supérieure de Lyon, 69007, Lyon, France

e-mail: alain.pumir@ens-lyon.fr

M. Wilkinson

Department of Mathematics and Statistics, The Open University, Walton Hall, Milton Keynes, MK7 6AA, UK

e-mail: m.wilkinson@open.ac.uk
} 
occurred by chance or by design [4]. In the context of fluid turbulence, models for the evolution of shapes of triangles [1-3] and tetrahedra [7-10] have been studied in the past decade in the fluid literature. The problem is strongly related to the scaling properties of a passive scalar advected by a turbulent velocity field [11-14]. In this context, the correlation functions and scaling exponents are naturally interpreted as the excited state of the evolution operator for the shape of a triangle [15]. Although we are focusing here on the ground state, technical similarities can be found with previous work on the problem of advection of a scalar by a smooth velocity field [16-18]. Our objective here is to define and analyse the motion of triplets of points in a simple model of turbulent flows. We obtain an exact expression for the steady-state statistics of the triangle shapes.

Our model flow is motivated by considering a scale decomposition of turbulent velocity fields. The flow at scales much larger than the size of the triangle merely translates and rotates the three points of the triangle, without any shape modification. Structures existing at a scale comparable to triangle size are modelled by applying a random matrix of unit determinant, to respect the incompressibility constraint. We refer to this motion as random strain (although the motion involves both strain and vorticity). Lastly, there may also be small-scale components in the flow which result in apparently uncorrelated motion of particles. We solve a model incorporating these effects, which is a simplification of a model introduced in $[1,7]$. Specifically, it is assumed that the particles evolve under the combined influence of a random strain (delta-correlated in time) and of independent Brownian motions. Because the displacement caused by a uniform strain is proportional to the scale size of the triangle, the strain will become dominant in the long-time limit as the separation of the particles grows. We therefore analyse a model where the Brownian displacement scales with the radius of gyration of the triangle. That is, we analyse a model for the small displacements $\delta \boldsymbol{x}_{i}$ of three particles with positions $\boldsymbol{x}_{i}$ which has the following structure:

$$
\delta \boldsymbol{x}_{i}=\delta \mathbf{A} \cdot \boldsymbol{x}_{i}+R \delta \boldsymbol{\xi}_{i},
$$

where $\delta \mathbf{A}$ is a traceless random symmetric matrix with diffusive fluctuations, $\delta \boldsymbol{\xi}_{i}$ is a Brownian displacement, and $R$ is the radius of gyration, defined by

$$
R^{2}=\frac{1}{6} \sum_{i=1}^{3} \sum_{j=1}^{3}\left|\boldsymbol{x}_{i}-\boldsymbol{x}_{j}\right|^{2} .
$$

The stochastic increments $\delta \mathbf{A}$ and $\delta \boldsymbol{x}_{i}$ in Eq. (1) are both multiplied by terms which depend upon the coordinates $\boldsymbol{x}_{i}$. This implies a potential source of ambiguity about how the increment $\delta \boldsymbol{x}_{i}$ at each time step should be evaluated [19]: do we evaluate the multiplier at the start of the step (the Itō convention) or at its mid-point (the Stratonovich convention)? However, we will show later that the ambiguity only affects the way the size of the triangle grows in time. When considering the evolution of the shape of the triangle, we do not need to distinguish whether the stochastic increments are of Itō or Stratonovich type.

In this article, we begin by investigating separately the action of the strain and of the Brownian terms. We then consider the two terms together, and find an exact expression for the distribution of the shapes of the triangles which are generated by this random process.

Specifying the shape of a triangle requires two parameters, which could be two of the three angles. For our purposes, however, another parametrisation is much more useful. D.G. Kendall argued that it is natural to describe the shape of a triangle by a point on the surface of a sphere [4], which we will refer to as the Kendall sphere. His paper also states that if the vertices of a triangle execute independent Brownian motions, then the image of this process 
is uniform diffusion on the surface of the Kendall sphere. His original paper [4] does not give a derivation of this result, and derivations which we have seen in later works rely upon computer algebra packages [6] or upon quite abstract machinery [5]. In Sect. 2 we start by explaining the Kendall sphere, its relation to other parameterisations, and we describe a Langevin equation which will prove to be a convenient representation of diffusion on the surface of a sphere. In Sect. 3 we define the ensemble of random strain $\delta \mathbf{A}$ introduced in (1), and evaluate the image of this random strain process on the surface of the Kendall sphere. We show that this leads to a diffusion process which is axially symmetric on the sphere. In Sect. 4 we use the same framework to give a direct demonstration of Kendall's result that the image of Brownian motion of the three points in Cartesian space is unbiased, isotropic and homogeneous diffusion on the Kendall sphere.

In Sect. 5 we combine the results of Sects. 3 and 4 to determine a Fokker-Planck equation which determines the probability density for the model (1) on the Kendall sphere. We solve this equation for the steady-state shape distribution.

The analysis in Sects. 3-5 uses the strategy of determining stochastic increments of polar coordinates on the Kendal sphere, and using these to surmise the Fokker-Planck equation. A complementary approach is to develop a Fokker-Planck equation for the model (1) directly, and make a change of variable. This approach is explored in Sect. 6, where we also show how the Fokker-Planck operator can be expressed as a linear combination of Casimir operators. Last, we briefly summarize our results in Sect. 7.

\section{The Kendall Sphere}

Consider a triangle in the plane formed by three points $\boldsymbol{x}_{i}, i=1,2,3$. The shape of the triangle is described by two two-component vectors $\boldsymbol{u}_{1}$ and $\boldsymbol{u}_{2}$ and by a $2 \times 2$ matrix w, defined by

$$
u_{1}=\frac{1}{\sqrt{2}}\left(x_{2}-x_{1}\right), \quad u_{2}=\frac{1}{\sqrt{6}}\left(2 x_{3}-x_{1}-x_{2}\right)
$$

and

$$
\mathbf{w}=\left(\begin{array}{ll}
u_{1,1} & u_{2,1} \\
u_{1,2} & u_{2,2}
\end{array}\right)
$$

where $u_{i, a}, a=1,2$ are the Cartesian components of $\boldsymbol{u}_{i}$. It is natural to represent the matrix $\mathbf{w}$ by a singular-value decomposition, writing

$$
\mathbf{w}=\mathbf{R}(\chi) \operatorname{diag}\left(\lambda_{1}, \lambda_{2}\right) \mathbf{R}(\phi / 2)
$$

where $\mathbf{R}(\theta)$ is the matrix describing a rotation by an angle $\theta$ :

$$
\mathbf{R}(\theta)=\left(\begin{array}{cc}
\cos \theta & -\sin \theta \\
\sin \theta & \cos \theta
\end{array}\right) .
$$

Note that the angle $\chi$ in (5) describes an overall rotation of the triangle, and is irrelevant to describing its shape. In this work the radius of gyration of the triangle $R$ is defined by:

$$
R^{2}=\sum_{i=1}^{3}\left|\boldsymbol{x}_{i}-\overline{\boldsymbol{x}}\right|^{2}
$$


and we find:

$$
R^{2}=\boldsymbol{u}_{1}^{2}+\boldsymbol{u}_{2}^{2}=\operatorname{tr}\left(\mathbf{w}^{\mathrm{T}} \mathbf{w}\right)=\lambda_{1}^{2}+\lambda_{2}^{2},
$$

where $\overline{\boldsymbol{x}}$ is the centre of mass of the three points. It is convenient to introduce a variable

$$
\zeta=\operatorname{det}(\mathbf{w})=\lambda_{1} \lambda_{2}
$$

which is proportional to the area $A$ of the triangle: $A=\sqrt{3} \zeta / 2$. We note that the size of the triangle can be characterized either by $R$ or by $\zeta$. Using $\zeta$ is natural when considering area-preserving dynamics [12]. However, because the parametrization involving $\zeta$ has a singularity when the three points defining the triangle become co-linear, it is more natural to use $R$ instead of $\zeta$ when the dynamics allows the vertices to become co-linear. For this reason we prefer $R$ over $\zeta$ as the scale coordinate throughout most of this paper. The angle $\phi$ characterizes the rotation in the space of triangles with a given set of values of $\lambda_{1}$ and $\lambda_{2}$. We note that a cyclic permutation of the vertices $\boldsymbol{x}_{i},(1,2,3) \rightarrow(2,3,1)$ (respectively $(1,2,3) \rightarrow(3,1,2))$ amounts to a transformation $\phi / 2 \rightarrow \phi / 2+2 \pi / 3$ (respectively $\phi / 2 \rightarrow$ $\phi / 2+4 \pi / 3)$.

It is useful to consider the form of the matrix $\mathbf{w}^{\mathrm{T}} \mathbf{w}$ :

$$
\begin{aligned}
\mathbf{w}^{\mathrm{T}} \mathbf{w} & =\left(\begin{array}{cc}
\boldsymbol{u}_{1}^{2} & \boldsymbol{u}_{1} \cdot \boldsymbol{u}_{2} \\
\boldsymbol{u}_{1} \cdot \boldsymbol{u}_{2} & \boldsymbol{u}_{2}^{2}
\end{array}\right) \\
& =\mathbf{R}(-\phi / 2) \operatorname{diag}\left(\lambda_{1}, \lambda_{2}\right) \mathbf{R}(-\chi) \mathbf{R}(\chi) \operatorname{diag}\left(\lambda_{1}, \lambda_{2}\right) \mathbf{R}(\phi / 2) \\
& =\mathbf{R}(-\phi / 2) \operatorname{diag}\left(\lambda_{1}^{2}, \lambda_{2}^{2}\right) \mathbf{R}(\phi / 2) \\
& =\left(\begin{array}{cc}
\frac{\lambda_{1}^{2}+\lambda_{2}^{2}}{2}+\frac{\lambda_{1}^{2}-\lambda_{2}^{2}}{2} \cos \phi & -\frac{\lambda_{1}^{2}-\lambda_{2}^{2}}{2} \sin \phi \\
-\frac{\lambda_{1}^{2}-\lambda_{2}^{2}}{2} \sin \phi & \frac{\lambda_{1}^{2}+\lambda_{2}^{2}}{2}-\frac{\lambda_{1}^{2}-\lambda_{2}^{2}}{2} \cos \phi
\end{array}\right)
\end{aligned}
$$

so that the geometrical properties of the vectors $\boldsymbol{u}_{1}$ and $\boldsymbol{u}_{2}$ have period $2 \pi$ in the angle $\phi$. With the formulation above, (10), it is easy to see that, with the extra convention that $\lambda_{1}^{2} \leq \lambda_{2}^{2}$, there is a one-to-one correspondence between the matrix $\mathbf{w}^{T} \mathbf{w}$ and a triplet of values $\left(\lambda_{1}^{2}, \lambda_{2}^{2}, \phi\right)$, with $0 \leq \phi \leq 2 \pi$. The relative sign of $\lambda_{1}$ and $\lambda_{2}$ is given by the condition (9). There are two possible overall signs for the values of $\lambda_{1}$ and $\lambda_{2}$. We note in this respect that the parametrization (5) is invariant under: $\left(\lambda_{1}, \lambda_{2}, \phi, \chi\right) \rightarrow\left(-\lambda_{1},-\lambda_{2}, \phi, \chi+\pi\right)$.

The shape of the triangle is fully determined by the variables $\phi$ and

$$
\Lambda=\frac{\lambda_{1}}{\lambda_{2}}
$$

In fact, the matrix $\mathbf{w} \cdot \mathbf{w}^{T}$ is expressed as:

$$
\mathbf{w}^{\mathrm{T}} \mathbf{w}=\lambda_{2}^{2}\left(\begin{array}{cc}
\frac{\Lambda^{2}+1}{2}+\frac{\Lambda^{2}-1}{2} \cos \phi & -\frac{\Lambda^{2}-1}{2} \sin \phi \\
-\frac{\Lambda^{2}-1}{2} \sin \phi & \frac{\Lambda^{2}+1}{2}-\frac{\Lambda^{2}-1}{2} \cos \phi
\end{array}\right),
$$

where the matrix in (12), which depends on the dimensionless variables $\Lambda$ and $\phi$, characterizes the shape of the triangle, and $\lambda_{2}^{2}$ its overall size. It is convenient to transform the ratio $\Lambda$ into another variable, $z$, defined by

$$
z=\frac{2 \operatorname{det}(\mathbf{w})}{\operatorname{tr}\left(\mathbf{w}^{\mathrm{T}} \mathbf{w}\right)}=\frac{2 \Lambda}{1+\Lambda^{2}}=\frac{2 \zeta}{R^{2}} .
$$


Note that the variable $z$, denoted $w$ in $[1,12]$, satisfies $-1 \leq z \leq+1$, so that a point in the region $|z| \leq 1,0 \leq \phi<2 \pi$ uniquely defines the shape of a triangle. The points $z= \pm 1$ correspond to equilateral triangles, with their shape being independent of the angular coordinate $\phi$. We can therefore regard the space of triangles as being equivalent to the surface of a sphere with azimuthal angle $\phi$, and with polar angle $\theta$, related to $z$ by $z=\cos \theta$. The equator corresponds to degenerate triangles consisting of three colinear points. The poles are equilateral triangles. There are three singular points on the equator, at which two points of the co-linear denerate triangle coincide. This device of representing the shape of a triangle by a point on the surface of a sphere was first introduced by D.G. Kendall [4]. We will refer to it as the Kendall sphere.

In the case where a triangle is subjected to a sequence of uncorrelated infinitesimal perturbations, the representative point diffuses over the surface of the sphere. We will characterise this diffusion process by specifying the stochastic increments $\delta z$ and $\delta \phi$ of the coordinates $z$ and $\phi$. In general the diffusion process may be inhomogeneous, it may have an anisotropic diffusion coefficient, and it may have drift terms. As a reference model, let us consider the case of homogeneous and isotropic diffusion on the Kendall sphere, with diffusion coefficient $D$. We characterise this process in terms of the stochastic increments of $z$ and $\phi$.

To characterise this homogeneous diffusive evolution let us use a system of standard Cartesian coordinates: $(x, y, z)=(\sin \theta \cos \phi, \sin \theta \sin \phi, \cos \theta)$. Without loss of generality we can use invariance with respect to the azimuthal angle $\phi$, and consider a point where $y=0$. Consider an orthogonal basis defined at the point $(\sin \theta, 0, \cos \theta)$ :

$$
\begin{aligned}
\mathbf{n} & =(\sin \theta, 0, \cos \theta)=(x, 0, z) \\
\mathbf{m} & =(-\cos \theta, 0, \sin \theta)=(-z, 0, x) \\
\mathbf{k} & =(0,1,0) .
\end{aligned}
$$

The diffusive fluctuation of a point $\mathbf{n}=(x, 0, z)$ on the surface is

$$
\delta \mathbf{n}=\delta X \mathbf{m}+\delta Y \mathbf{k}+\delta Z \mathbf{n},
$$

where $\delta X$ and $\delta Y$ are angular increments, satisfying $\langle\delta X\rangle=\langle\delta Y\rangle=0$ and

$$
\left\langle\delta X^{2}\right\rangle=\left\langle\delta Y^{2}\right\rangle=2 D \delta t, \quad\langle\delta X \delta Y\rangle=0 .
$$

The value of $\delta Z$ is determined from $\delta X$ and $\delta Y$ using the constraint that $\mathbf{n}$ remains normalised: this implies that $\delta Z=-\frac{1}{2}\left(\delta X^{2}+\delta Y^{2}\right)$. Consider the increments of $z$ and $\phi$ under this process: we have $\delta Y=\sin \theta \delta \phi$ and $(\mathbf{n}+\delta \mathbf{n}) \cdot \mathbf{e}_{3}=z+\delta z=\cos \theta-\sin \theta \delta X+\cos \theta \delta Z$. Solving for $\delta \phi, \delta z$, we find

$$
\begin{aligned}
\delta \phi & =\frac{1}{x} \delta Y \\
\delta z & =-x \delta X-\frac{1}{2} z\left(\delta X^{2}+\delta Y^{2}\right)
\end{aligned}
$$

where $z=\cos \theta, x=\sqrt{1-z^{2}}$. The diffusion and drift coefficients for homogeneous diffusion on the sphere, defined by writing $\langle\delta z\rangle=v_{z} \delta t,\left\langle\delta z^{2}\right\rangle=2 D_{z} \delta t,\left\langle\delta \phi^{2}\right\rangle=2 D_{\phi} \delta t$, are 
therefore

$$
\begin{aligned}
D_{z} & =\left(1-z^{2}\right) D \\
D_{\phi} & =\frac{D}{1-z^{2}} \\
v_{z} & =-2 z D \\
v_{\chi} & =0 .
\end{aligned}
$$

This representation of homogeneous, isotropic diffusion on the surface of a sphere will prove to be an instructive comparison case for the random strain model which we consider next.

\section{Image of Random Strain on the Kendall Sphere}

In this section, we consider the action of three vertices $\boldsymbol{r}_{i}$ under the action of a random strain. Using the parametrization (4) of the shape of the triangle in terms of the matrix $\mathbf{w}$, the equation of evolution over a finite time $\delta t$ is written as:

$$
\delta \mathbf{w}=\delta \mathbf{A} \cdot \mathbf{w},
$$

where $\delta \mathbf{A}$ is an infinitesimal random strain, of the form

$$
\delta \mathbf{A}=\left(\begin{array}{cc}
\delta a & \delta b \\
\delta b & -\delta a
\end{array}\right)
$$

In the limit $\delta t \rightarrow 0$, (19) corresponds to a Langevin equation for the matrix $\mathbf{w}$, which evolves under the action of a strain matrix, $\delta$-correlated in time. The statistics of the elements of the random strain $\delta \mathbf{A}$ in (20) are $\langle\delta a\rangle=\langle\delta b\rangle=0$ and

$$
\left\langle\delta a^{2}\right\rangle=2 D_{a} \delta t, \quad\left\langle\delta b^{2}\right\rangle=2 D_{b} \delta t, \quad\langle\delta a \delta b\rangle=0 .
$$

Imposing the requirement that the statistics of this random strain matrix are rotationally invariant leads to the condition $D_{a}=D_{b} \equiv D_{s}$. An infinitesimal rotation could have been included in the definition of the random strain (by making the off-diagonal elements dissimilar), but this is clearly irrelevant to shape fluctuations.

The strain term, introduced in (19), acts multiplicatively on the variables defining the shape $\mathbf{w}$, so in a time $\delta t$, the matrix $\mathbf{w}$ is transformed into $\mathbf{w}^{\prime}$ according to:

$$
\mathbf{w}^{\prime}=(\mathbf{I}+\delta \mathbf{A}) \mathbf{w}=\mathbf{R}(\chi+\delta \chi) \operatorname{diag}\left(\lambda_{1}+\delta \lambda_{1}, \lambda_{2}+\delta \lambda_{2}\right) \mathbf{R}(\phi / 2+\delta \phi / 2),
$$

where we have used the decomposition (5) to parametrize $\mathbf{w}$, and $\delta \mathbf{A}$ is the infinitesimal random strain defined by (20). Because the random strain acts multiplicatively on the shape of the triangle, there is in fact an ambiguity in determining the evolution of $\mathbf{w}$ in (22); the precise definition of $\delta \mathbf{A} \cdot \mathbf{w}$ matters when practically integrating the equations. The simplest procedure consisting in taking $\mathbf{w}(t+\delta t)-\mathbf{w}(t) \approx \delta \mathbf{A} \cdot \mathbf{w}(t)$ (the Itō procedure), and the physically more correct procedure, consisting in defining: $\mathbf{w}(t+\delta t)-\mathbf{w}(t) \approx \frac{1}{2} \delta \mathbf{A} \cdot(\mathbf{w}(t)+$ $\mathbf{w}(t+\delta t)$ ) (the Stratonovich procedure) [19] differ by a term of the form $\frac{1}{2} \delta \mathbf{A}^{2}$, whose mean value is a diagonal term, equal to $2 D_{s} \delta t \mathbf{I}$, which dilates in a similar way the two eigenvalues $\lambda_{1}$ and $\lambda_{2}$, thus leaving the variables that characterize the shape, $z$ and $\phi$, unaffected. For this 
reason, the precise way of defining the model here turns out to be immaterial for determining the shape fluctuations.

Consider the evolution of the parameters $\phi, \chi, \lambda_{1}, \lambda_{2}$ under this random strain evolution: from (22) we obtain

$$
\mathbf{R}(\delta \chi) \operatorname{diag}\left(\lambda_{1}+\delta \lambda_{1}, \lambda_{2}+\delta \lambda_{2}\right) \mathbf{R}(\delta \phi / 2)=\operatorname{diag}\left(\lambda_{1}, \lambda_{2}\right)+\delta \mathbf{A}^{\prime} \operatorname{diag}\left(\lambda_{1}, \lambda_{2}\right),
$$

where

$$
\delta \mathbf{A}^{\prime}=\mathbf{R}(-\chi) \delta \mathbf{A} \mathbf{R}(\chi) \equiv\left(\begin{array}{cc}
\delta a^{\prime} & \delta b^{\prime} \\
\delta b^{\prime} & -\delta a^{\prime}
\end{array}\right) .
$$

In the following we use the rotational invariance of the statistics of $\delta \mathbf{A}$ to conclude that the statistics of its elements satisfy $\left\langle\delta a^{\prime 2}\right\rangle=\left\langle\delta b^{\prime 2}\right\rangle=2 D_{s} \delta t$. Expanding the factors of Eq. (23) up to quadratic terms in the small increments $\delta \chi, \delta \phi$ and $\delta \lambda_{i}$, and collecting terms, we find

$$
\begin{aligned}
\lambda_{1} \delta a^{\prime} & =\delta \lambda_{1}-\frac{\lambda_{1}}{8}\left(4 \delta \chi^{2}+\delta \phi^{2}\right)-\frac{\lambda_{2}}{2} \delta \chi \delta \phi \\
-\lambda_{2} \delta a^{\prime} & =\delta \lambda_{2}-\frac{\lambda_{2}}{8}\left(4 \delta \chi^{2}+\delta \phi^{2}\right)-\frac{\lambda_{1}}{2} \delta \chi \delta \phi \\
\lambda_{2} \delta b^{\prime} & =\lambda_{2} \delta \chi+\frac{\lambda_{1}}{2} \delta \phi+\frac{1}{2} \delta \lambda_{1} \delta \phi+\delta \lambda_{2} \delta \chi \\
\lambda_{1} \delta b^{\prime} & =-\lambda_{1} \delta \chi-\frac{\lambda_{2}}{2} \delta \phi-\delta \lambda_{1} \delta \chi-\frac{1}{2} \delta \lambda_{2} \delta \phi .
\end{aligned}
$$

The first order relations between the increments $\delta a^{\prime}, \delta b^{\prime}$ and the increments of the shape parameters are therefore

$$
\begin{aligned}
\delta \lambda_{1} & =\lambda_{1} \delta a^{\prime} \\
\delta \lambda_{2} & =-\lambda_{2} \delta a^{\prime} \\
\delta \phi & =\frac{4 \lambda_{1} \lambda_{2}}{\lambda_{1}^{2}-\lambda_{2}^{2}} \delta b^{\prime} \\
\delta \chi & =\frac{\lambda_{1}^{2}+\lambda_{2}^{2}}{\lambda_{2}^{2}-\lambda_{1}^{2}} \delta b^{\prime}
\end{aligned}
$$

so that

$$
\begin{aligned}
\left\langle\delta \chi^{2}\right\rangle & =\frac{2 D_{s} \delta t\left(\lambda_{1}^{2}+\lambda_{2}^{2}\right)^{2}}{\left(\lambda_{1}^{2}-\lambda_{2}^{2}\right)^{2}} \\
\left\langle\delta \phi^{2}\right\rangle & =\frac{32 D_{s} \delta t \lambda_{1}^{2} \lambda_{2}^{2}}{\left(\lambda_{1}^{2}-\lambda_{2}^{2}\right)^{2}} \\
\langle\delta \phi \delta \chi\rangle & =\frac{-8 D_{s} \delta t \lambda_{1} \lambda_{2}\left(\lambda_{1}^{2}+\lambda_{2}^{2}\right)}{\left(\lambda_{1}^{2}-\lambda_{2}^{2}\right)^{2}} .
\end{aligned}
$$


Adding the corrections arising from the quadratic terms then gives

$$
\begin{aligned}
\lambda_{1} \delta a^{\prime} & =\delta \lambda_{1}-\lambda_{1} \frac{\lambda_{1}^{2}+3 \lambda_{2}^{2}}{\lambda_{1}^{2}-\lambda_{2}^{2}} D_{s} \delta t \\
-\lambda_{2} \delta a^{\prime} & =\delta \lambda_{2}+\lambda_{2} \frac{\lambda_{2}^{2}+3 \lambda_{1}^{2}}{\lambda_{1}^{2}-\lambda_{2}^{2}} D_{s} \delta t \\
\lambda_{2} \delta b^{\prime} & =\lambda_{2} \delta \chi+\frac{1}{2} \lambda_{1} \delta \phi \\
\lambda_{1} \delta b^{\prime} & =-\lambda_{1} \delta \chi-\frac{1}{2} \lambda_{2} \delta \phi .
\end{aligned}
$$

The first two of these relations are consistent with the constraint $\delta\left(\lambda_{1} \lambda_{2}\right)=0$, and the second pair follow from $\left\langle\delta a^{\prime} \delta b^{\prime}\right\rangle=0$.

Now transform to new variables $\Lambda=\lambda_{1} / \lambda_{2}, R=\sqrt{\lambda_{1}^{2}+\lambda_{2}^{2}}$. It is clear that the evolution is scale invariant, so that the dynamics does not depend upon the radius of gyration $R$. We find

$$
\begin{aligned}
\delta \Lambda & =\frac{\delta \lambda_{1}}{\lambda_{2}}-\Lambda \frac{\delta \lambda_{2}}{\lambda_{2}}+\Lambda \frac{\delta \lambda_{2}^{2}}{\lambda_{2}^{2}}-\frac{\delta \lambda_{1} \delta \lambda_{2}}{\lambda_{2}^{2}} \\
& =2 \Lambda \delta a^{\prime}+\frac{8 \lambda_{1}^{2} \Lambda D_{s} \delta t}{\lambda_{1}^{2}-\lambda_{2}^{2}} .
\end{aligned}
$$

The increment of $\phi$ is

$$
\delta \phi=\frac{4 \lambda_{1} \lambda_{2}}{\lambda_{1}^{2}-\lambda_{2}^{2}} \delta b^{\prime}=\frac{4 \Lambda}{\Lambda^{2}-1} \delta b^{\prime} .
$$

The drift and diffusion coefficients in the $\Lambda, \phi$ variables are:

$$
\begin{aligned}
D_{\Lambda}(\Lambda) & =4 \Lambda^{2} D_{s} \\
D_{\phi}(\Lambda) & =\frac{16 \Lambda^{2} D_{s}}{\left(\Lambda^{2}-1\right)^{2}} \\
v_{\Lambda}(\Lambda) & =\frac{8 \Lambda^{3} D_{s}}{\Lambda^{2}-1} \\
v_{\phi} & =0 .
\end{aligned}
$$

Now work in terms of $z=2 \Lambda /\left(\Lambda^{2}+1\right)$ : the diffusion and drift coefficients are

$$
\begin{gathered}
D_{z}(\Lambda)=\left(\frac{\mathrm{d} z}{\mathrm{~d} \Lambda}\right)^{2} D_{\Lambda}=\frac{4\left(1-\Lambda^{2}\right)^{2}}{\left(1+\Lambda^{2}\right)^{4}} 4 \Lambda^{2} D_{s} \\
v_{z}(\Lambda)=\frac{\mathrm{d} z}{\mathrm{~d} \Lambda} v_{\Lambda}+\frac{\mathrm{d}^{2} z}{\mathrm{~d} \Lambda^{2}} D_{s} \Lambda=\frac{-64 D_{s} \Lambda^{3}}{\left(\Lambda^{2}+1\right)^{3}} .
\end{gathered}
$$

To express these in terms of $z$, consider an intermediate variable $\Theta$, satisfying

$$
\Lambda=\frac{\sin \Theta}{\cos \Theta}, \quad z=\sin (2 \Theta) .
$$


Then:

$$
\begin{aligned}
D_{z}(z) & =\frac{16 D_{s} \Lambda^{2}\left(1-\Lambda^{2}\right)^{2}}{\left(1+\Lambda^{2}\right)^{4}} \\
& =4 D_{s} \sin ^{2}(2 \Theta) \cos ^{2}(2 \Theta) \\
& =4 D_{s} z^{2}\left(1-z^{2}\right)
\end{aligned}
$$

and

$$
\begin{aligned}
D_{\phi}(z) & =\frac{16 \Lambda^{2} D_{s}}{\Lambda^{2}-1}=-\frac{4 D_{s} \sin ^{2}(2 \Theta)}{\cos ^{2}(2 \Theta)} \\
& =\frac{4 D_{s} z^{2}}{1-z^{2}}
\end{aligned}
$$

finally

$$
\begin{aligned}
v_{z}(z) & =\frac{-32 D_{s} \Lambda^{3}}{\left(1+\Lambda^{2}\right)^{3}} \\
& =-8 D_{s} z^{3} .
\end{aligned}
$$

It is interesting to note that the coefficients differ from those of the homogeneous diffusion on a sphere (considered in Sect. 2) by a simple factor, namely $4 z^{2}$.

\section{Image of Brownian Motion on the Kendall Sphere}

We consider in this section the action of a random Brownian motion on the vertices of the triangle. Specifically, let the vertices of a triangle make small, independent and isotropic diffusive displacements, as if they are undergoing Brownian motion:

$$
\delta x_{i, a}=\delta \xi_{i, a},
$$

where the vectors $\delta \xi_{i, a}$ satisfy:

$$
\left\langle\delta \xi_{i, j}\right\rangle=0, \quad\left\langle\delta \xi_{i, j} \delta \xi_{k, l}\right\rangle=2 \delta_{i k} \delta_{j l} D_{b} \delta t
$$

where $D_{b}$ is a diffusion coefficient.

In the Introduction we mentioned that if the vertices execute independent Brownian motion, then the dynamics of the representative point on the surface of the Kendall sphere is homogeneous diffusion. The published derivations [5, 6] are difficult to compare with the derivation of the diffusion process for the random strain model which we analysed in Sect. 3. In this section we present an elementary derivation of Kendall's result, using the same approach as for the random strain model.

As in the previous section, we characterise the change in the triangle by considering a change of the matrix $\mathbf{w}$ :

$$
\delta \mathbf{w}=\delta \eta
$$

with:

$$
\delta \eta_{1, a}=\frac{1}{\sqrt{2}}\left(\delta \xi_{2, a}-\delta \xi_{1, a}\right), \quad \delta \eta_{2, a}=\frac{1}{\sqrt{6}}\left(2 \delta \xi_{3, a}-\delta \xi_{1, a}-\delta \xi_{2, a}\right) .
$$


The statistics of these small displacements satisfy

$$
\left\langle\delta \eta_{i, j}\right\rangle=0, \quad\left\langle\delta \eta_{i, j} \delta \eta_{k, l}\right\rangle=2 \delta_{i k} \delta_{j l} D_{b} \delta t .
$$

Note that $\left\langle\delta \eta_{i, j}^{2}\right\rangle=\left\langle\delta \xi_{k, l}^{2}\right\rangle$ for any choice of $i, j, k, l$, so that $D_{b}$ is the same as the diffusion coefficient for the Brownian motion in coordinate space.

Using the parametrization defined by (5), (39) leads to a change of $\lambda_{1}, \lambda_{2}$ and $\phi$, hence a change in the variables $\phi$ and $z$. Applying rotation matrices to $\mathbf{w}+\delta \mathbf{w}$, we obtain

$$
\begin{aligned}
\mathbf{R}(-\chi)(\mathbf{w}+\delta \mathbf{w}) \mathbf{R}(-\phi / 2) & =\operatorname{diag}\left(\lambda_{1}, \lambda_{2}\right)+\mathbf{R}(-\chi)\left(\begin{array}{ll}
\delta \eta_{1,1} & \delta \eta_{2,1} \\
\delta \eta_{1,2} & \delta \eta_{2,2}
\end{array}\right) \mathbf{R}(-\phi / 2) \\
& \equiv \operatorname{diag}\left(\lambda_{1}, \lambda_{2}\right)+\delta \eta^{\prime} .
\end{aligned}
$$

The statistics of the components $\delta \eta^{\prime}$, where the $\delta \eta_{i, j}^{\prime}$ are components of $\delta \eta_{i, j}$ in the rotated coordinate system, are independent of $\chi$ and $\phi$ : for example

$$
\begin{aligned}
\delta \eta_{11}^{\prime}= & \cos \chi \cos (\phi / 2) \delta \eta_{1,1}-\sin \chi \cos (\phi / 2) \delta \eta_{1,2}+\cos \chi \sin (\phi / 2) \delta \eta_{2,1} \\
& -\sin \chi \sin (\phi / 2) \delta \eta_{2,2}
\end{aligned}
$$

has a variance $\left\langle\left(\delta \eta_{11}^{\prime}\right)^{2}\right\rangle=\left\langle\left(\delta \eta_{11}\right)^{2}\right\rangle$ which is independent of $\phi$ and $\chi$, and the same is true of the other components of $\delta \eta_{i j}$. Because of this invariance it suffices to perform perturbation theory about the $\chi=0, \phi=0$ case:

$$
\begin{aligned}
\left(\begin{array}{cc}
\lambda_{1}+\delta \eta_{1,1}^{\prime} & \delta \eta_{2,1}^{\prime} \\
\delta \eta_{1,2}^{\prime} & \lambda_{2}+\delta \eta_{2,2}^{\prime}
\end{array}\right)= & \left(\begin{array}{cc}
\cos \delta \chi & \sin \delta \chi \\
-\sin \delta \chi & \cos \delta \chi
\end{array}\right)\left(\begin{array}{cc}
\lambda_{1}+\delta \lambda_{1} & 0 \\
0 & \lambda_{2}+\delta \lambda_{2}
\end{array}\right) \\
& \times\left(\begin{array}{cc}
\cos (\delta \phi / 2) & \sin (\delta \phi / 2) \\
-\sin (\delta \phi / 2) & \cos (\delta \phi / 2)
\end{array}\right)
\end{aligned}
$$

Expanding in powers of $\delta \theta$ and $\delta \chi$, and retaining terms only up to quadratic order in the small increments, we find

$$
\begin{aligned}
& \delta \eta_{1,1}^{\prime}=\delta \lambda_{1}-\frac{\lambda_{1}}{8}\left(4 \delta \chi^{2}+\delta \phi^{2}\right)-\frac{\lambda_{2}}{2} \delta \chi \delta \phi \\
& \delta \eta_{2,2}^{\prime}=\delta \lambda_{2}-\frac{\lambda_{2}}{8}\left(4 \delta \chi^{2}+\delta \phi^{2}\right)-\frac{\lambda_{1}}{2} \delta \chi \delta \phi \\
& \delta \eta_{1,2}^{\prime}=-\lambda_{1} \delta \chi-\frac{\lambda_{2}}{2} \delta \phi-\frac{\delta \lambda_{2} \delta \phi}{2}-\delta \lambda_{1} \delta \chi \\
& \delta \eta_{2,1}^{\prime}=\frac{\lambda_{1}}{2} \delta \phi+\lambda_{2} \delta \chi+\frac{\delta \lambda_{1} \delta \phi}{2}+\delta \lambda_{2} \delta \chi .
\end{aligned}
$$

These equations can be solved to determine $\delta \lambda_{i}, \delta \theta$ and $\delta \chi$ in terms of the elements of $\delta \eta^{\prime}$. We follow the same approach as in Sect. 3. First, compute the linear terms from the linear terms of (45). We then use these expressions to estimate the covariances of the small 
increments $\delta \phi, \delta \chi$ and $\delta \lambda_{i}$. We find that the stochastic increments are

$$
\begin{aligned}
\delta \lambda_{1} & =\delta \eta_{1,1}^{\prime}+\frac{2 \lambda_{1} D_{b} \delta t}{\lambda_{1}^{2}-\lambda_{2}^{2}} \\
\delta \lambda_{2} & =\delta \eta_{2,2}^{\prime}-\frac{2 \lambda_{2} D_{b} \delta t}{\lambda_{1}^{2}-\lambda_{2}^{2}} \\
\delta \phi & =\frac{2\left(\lambda_{1} \delta \eta_{2,1}^{\prime}+\lambda_{2} \delta \eta_{1,2}^{\prime}\right)}{\lambda_{1}^{2}-\lambda_{2}^{2}} \\
\delta \chi & =\frac{\lambda_{1} \delta \eta_{1,2}^{\prime}+\lambda_{2} \delta \eta_{2,1}^{\prime}}{\lambda_{2}^{2}-\lambda_{1}^{2}}
\end{aligned}
$$

From now on we concentrate upon the evolution of the coordinates which define the shape, namely $\phi$ and $\Lambda=\lambda_{1} / \lambda_{2}$. Note that

$$
\delta \Lambda=\frac{\lambda_{1}+\delta \lambda_{1}}{\lambda_{2}+\delta \lambda_{2}}-\frac{\lambda_{1}}{\lambda_{2}}=\frac{\delta \lambda_{1}}{\lambda_{2}}-\Lambda \frac{\delta \lambda_{2}}{\lambda_{2}}+\Lambda \frac{\delta \lambda_{2}^{2}}{\lambda_{2}^{2}}-\frac{\delta \lambda_{1} \delta \lambda_{2}}{\lambda_{2}^{2}} .
$$

Now normalise the scale of the triangle so that the radius of gyration $\sqrt{\lambda_{1}^{2}+\lambda_{2}^{2}}=R$ is factored out of the stochastic increments:

$$
\begin{aligned}
\delta \Lambda & =\frac{\delta \eta_{1,1}^{\prime}}{\lambda_{2}}+\frac{2 \Lambda D_{b} \delta t}{\lambda_{1}^{2}-\lambda_{2}^{2}}-\frac{\Lambda \delta \eta_{2,2}^{\prime}}{\lambda_{2}}+\frac{2 \Lambda D_{b} \delta t}{\lambda_{1}^{2}-\lambda_{2}^{2}}+\frac{2 \Lambda D_{b} \delta t}{\lambda_{2}^{2}} \\
& =\frac{1}{R} \frac{\delta \eta_{1,1}^{\prime}}{\lambda_{2} R}-\frac{\Lambda}{R} \frac{\delta \eta_{2,2}^{\prime}}{\lambda_{2} R}+\frac{2 \Lambda D_{b} \delta t}{R^{2}}\left[\frac{2 R^{2}}{\lambda_{1}^{2}-\lambda_{2}^{2}}+\frac{R^{2}}{\lambda_{2}^{2}}\right] \\
& =\frac{1}{R} \frac{\delta \eta_{1,1}^{\prime}}{\lambda_{2} R}-\frac{\Lambda}{R} \frac{\delta \eta_{2,2}^{\prime}}{\lambda_{2} R}+\frac{1}{R^{2}} \frac{2 \Lambda\left(\Lambda^{2}+1\right)^{2} D_{b} \delta t}{\Lambda^{2}-1} .
\end{aligned}
$$

We consider the evolution of the shape of the triangle with the radius of gyration frozen. When the radius of gyration is $R=1$, the increment of $\Lambda$ may therefore be written

$$
\delta \Lambda=\left(1+\Lambda^{2}\right) \delta \gamma_{1}+\frac{2 \Lambda\left(1+\Lambda^{2}\right)^{2} D_{b} \delta t}{\left(\Lambda^{2}-1\right)},
$$

where

$$
\delta \gamma_{1}=\frac{\delta \eta_{1,1}^{\prime}-\Lambda \delta \eta_{2,2}^{\prime}}{\sqrt{1+\Lambda^{2}}}
$$

is a stochastic increment which satisfies $\left\langle\delta \gamma_{1}{ }^{2}\right\rangle=2 D_{b} \delta t,\left\langle\delta \gamma_{1}\right\rangle=0$. Similarly, the increment of $\phi$ may be expressed as

$$
\begin{aligned}
\delta \phi & =\frac{2 \sqrt{\Lambda^{2}+1}}{\lambda_{2}\left(\Lambda^{2}-1\right)} \delta \gamma_{2} \\
& =\frac{1}{R} \frac{2\left(\Lambda^{2}+1\right)}{\Lambda^{2}-1} \delta \gamma_{2},
\end{aligned}
$$


where

$$
\delta \gamma_{2}=\frac{\Lambda \delta \eta_{1,1}^{\prime}+\delta \eta_{1,2}^{\prime}}{\sqrt{\Lambda^{2}+1}}
$$

is a stochastic increment with the same statistics as $\delta \gamma_{1}$, that is $\left\langle\delta \gamma_{2}{ }^{2}\right\rangle=2 D_{b} \delta t$.

The shape fluctuations are described by fluctuations of just two variables, $\phi$ and $\Lambda$, which evolve diffusively. Their coefficients of diffusion and drift are independent of $\phi$ :

$$
\begin{aligned}
D_{\phi}(\Lambda) & =\frac{4\left(\Lambda^{2}+1\right)^{2}}{\left(\Lambda^{2}-1\right)^{2}} D_{b} \\
D_{\Lambda}(\Lambda) & =\left(\Lambda^{2}+1\right)^{2} D_{b} \\
v_{\Lambda}(\Lambda) & =\frac{2 \Lambda\left(\Lambda^{2}+1\right)^{2}}{\Lambda^{2}-1} D_{b} \\
v_{\phi} & =0 .
\end{aligned}
$$

An alternative coordinate is $z=2 \Lambda /\left(\Lambda^{2}+1\right)$. Expressing the stochastic increments in the $(z, \phi)$ coordinate system, we obtain the following expressions for the diffusion and drift coefficients:

$$
\begin{aligned}
D_{z} & =\left(\frac{\mathrm{d} z}{\mathrm{~d} \Lambda}\right)^{2} D_{\Lambda}=4\left(\frac{\Lambda^{2}-1}{\Lambda^{2}+1}\right)^{2} D_{b} \\
v_{z} & =\frac{\mathrm{d} z}{\mathrm{~d} \Lambda} v_{\Lambda}+\frac{\mathrm{d}^{2} z}{\mathrm{~d} \Lambda^{2}} D_{\Lambda}=\frac{-16 \Lambda}{\Lambda^{2}+1} D_{b} .
\end{aligned}
$$

Again following the approach in Sect. 3, we express the diffusion and drift coefficients in terms of $z$ by introducing a variable $\Theta$ defined by $\Lambda=\sin \Theta / \cos \Theta$, so that $z=\sin (2 \Theta)$. Now express the coefficients in terms of $z$, and obtain

$$
\begin{aligned}
& D_{z}(z)=4\left(\frac{1-\Lambda^{2}}{1+\Lambda^{2}}\right)^{2} D_{b}=\frac{4\left(\sin ^{2} \Theta-\cos ^{2} \Theta\right)^{2}}{\left(\sin ^{2} \Theta+\cos ^{2} \Theta\right)^{2}} D_{b}=4 \cos ^{2}(2 \Theta) D_{b}=4\left(1-z^{2}\right) D_{b} \\
& D_{\phi}(z)=4\left(\frac{\Lambda^{2}+1}{\Lambda^{2}-1}\right)^{2} D_{b}=\frac{16 D_{b}^{2}}{D_{z}(z)}=\frac{4 D_{b}}{1-z^{2}} \\
& v_{z}(z)=\frac{-16 \Lambda}{\Lambda^{2}+1} D_{b}=-8 z D_{b} .
\end{aligned}
$$

These are the same as for simple diffusion on a sphere, as discussed in Sect. 2, with the diffusion coefficient $D_{b}$ replaced by $4 D_{b}$. These coefficients are also very closely related to those obtained in the random strain model: the coefficients for that model all differ by a factor of $z^{2}$.

\section{Mixed Model}

Consider the distribution of triangle shapes for a mixed model, in which the displacement of the three particles in a short time interval $\delta t$ is a combination of a random strain and an 
uncorrelated random displacement, which is proportional to the radius of gyration $R$. This leads to the following map, using the notation of (1):

$$
\boldsymbol{x}_{i}^{\prime}=\boldsymbol{x}_{i}+\delta \boldsymbol{x}_{i}=(\mathbf{I}+\delta \mathbf{A}) \boldsymbol{x}_{i}+R \delta \boldsymbol{\xi}_{i}
$$

As we have done in the previous subsections, we express the evolution in terms of the matrix w, see (4), (5):

$$
\delta \mathbf{w}=\delta \mathbf{A} \cdot \mathbf{w}+R \delta \eta
$$

which combines (19) and (39), and describes the evolution of the shape of the triangle, from $\mathbf{w}$ to $\mathbf{w}^{\prime}=\mathbf{w}+\delta \mathbf{w}$ in a time $\delta t$. The statistics of the elements of $\delta \mathbf{A}$ are given by (21), and $\delta \boldsymbol{\eta}_{i}$ has a Gaussian distribution, characterized by $\left\langle\delta \boldsymbol{\eta}_{i}\right\rangle=0$ and $\left\langle\delta \boldsymbol{\eta}_{i} \cdot \delta \boldsymbol{\eta}_{j}\right\rangle=4 D_{\mathrm{b}} \delta_{i j} \delta t$, where $D_{\mathrm{b}}$ is the diffusion coefficient associated with Brownian motion. The scaling of the Brownian component in (56) implies that the increments of $\Lambda$ and $\phi$ due to the Brownian motion are given by Eqs. (49) and (51), alternatively by stochastic increments $\delta z, \delta \phi$ which can be expressed using the drift and diffusion coefficients (55). Similarly, there are independent contributions to $\delta z$ and $\delta \phi$ from the shearing motion. These are described by stochastic increments with drift and diffusion coefficients given by Eqs. (34), (35) and (36).

The stochastic terms $\delta \mathbf{A}$ and $\delta \boldsymbol{\eta}_{i}$ in (57) both act multiplicatively, either on the variables $\mathbf{w}$, or upon the radius of gyration $R$, which is a function of these variables. This could potentially result in an ambiguity in defining the model [19], depending upon whether we use the Itō or Stratonovich definition of the stochastic increment. We notice in this respect the ambiguity in the evolution defined by the Brownian term only affects the radial variable $R$, and not the shape variable. As pointed out in Sect. 3, the same is true for the shear term.

The fluctuations of shape are therefore described by a Fokker-Planck equation for the joint probability density on the Kendal sphere, $P(z, \phi, t)$

$$
\frac{\partial P}{\partial t}=-\frac{\partial}{\partial z}\left[v_{z}(z) P\right]+\frac{\partial^{2}}{\partial z^{2}}\left[D_{z}(z) P\right]+\frac{\partial^{2}}{\partial \phi^{2}}\left[D_{\phi}(z) P\right]
$$

with

$$
\begin{aligned}
v_{z}(z) & =-8 D_{\mathrm{b}} z-8 D_{\mathrm{s}} z^{3} \\
D_{z}(z) & =4\left(1-z^{2}\right) D_{\mathrm{b}}+4 z^{2}\left(1-z^{2}\right) D_{\mathrm{s}} \\
D_{\phi}(z) & =\frac{4 D_{\mathrm{b}}}{1-z^{2}}+\frac{4 D_{\mathrm{s}} z^{2}}{1-z^{2}} .
\end{aligned}
$$

The steady state solution only depends upon the ratio of diffusion coefficients

$$
\epsilon=\frac{D_{\mathrm{b}}}{D_{\mathrm{s}}}
$$

This has a steady state solution where $\phi$ is uniform on the circle and where the marginal distribution for $z$ satisfies

$$
2 z\left(\epsilon+z^{2}\right) P+\frac{\partial}{\partial z}\left[\left(\epsilon+z^{2}\right)\left(1-z^{2}\right) P\right]=0
$$

so that

$$
2 z P+\left(\epsilon+z^{2}\right) \frac{\partial P}{\partial z}=0 .
$$


Fig. 1 The PDF of $z$ for values of $\epsilon \equiv D_{\mathrm{b}} / D_{\mathrm{s}}, \epsilon=3 \times 10^{-4}$, $10^{-3}, 3 \times 10^{-3}, 10^{-2}$ and $3 \times 10^{-2}$, shown as a full line. The fit by Eq. (63) is shown as a dashed line

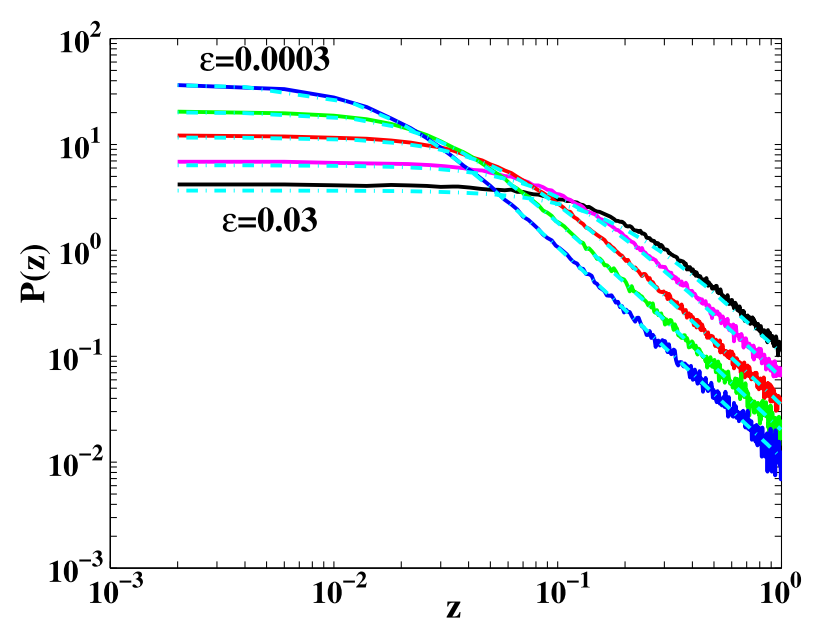

This has a solution

$$
P(z)=\frac{\sqrt{\epsilon}}{2 \tan ^{-1}(1 / \sqrt{\epsilon})} \frac{1}{\epsilon+z^{2}},
$$

where the multiplier normalises $P(z)$ on $[-1,1]$.

To check the prediction of (63), we have studied numerically the mixed model defined by (56). One of the crucial properties of the random strain is that it conserves exactly the area of the triangle: $\zeta$ is a constant. We stress that this constraint is satisfied only when the evolution equation (56) is treated using the Stratonovich convention [19]. One readily notices that the expression for the evolution operator, induced by the strain, and correct up to order $\delta t^{1}$, is simply:

$$
\boldsymbol{x}_{i}^{\prime}=\left(\mathbf{I}+\delta \mathbf{A}+\delta \mathbf{A}^{2} / 2\right) \boldsymbol{x}_{i}
$$

which is the expansion of the exponential of the traceless matrix $\delta \mathbf{A}$ up to second order. To impose strictly the constraint of area conservation, we replaced the operator in (64) by $\exp (\delta \mathbf{A})$, whose determinant is exactly equal to 1 . and has the correct expansion, up to terms of order $\delta t^{3 / 2}$. Using the fact that: $\delta \mathbf{A}^{2}=\left(\delta a^{2}+\delta b^{2}\right) \mathbf{I}$, one readily finds that

$$
\exp (\delta \mathbf{A})=\cosh \left(\sqrt{\left(\delta a^{2}+\delta b^{2}\right)}\right) \mathbf{I}+\frac{\sinh \left(\sqrt{\left(\delta \mathbf{a}^{2}+\delta \mathbf{b}^{2}\right)}\right)}{\sqrt{\left(\delta \mathbf{a}^{2}+\delta \mathbf{b}^{2}\right)}} \delta \mathbf{A}
$$

Also, the Brownian motion term acts in (1) multiplicatively (because of $R$ ). As we are interested here only in the shape evolution, and not on the evolution of the size, $R$, which requires some care, we have projected the term $R \delta \boldsymbol{r}_{i}$ term in the direction transverse to the radial direction, which does not affect the shape dynamics, but simplifies the numerical integration.

The data shown in Fig. 1 was obtained by iterating the mapping given by (56) for $3 \times 10^{4}$ time steps, over $4 \times 10^{5}$ different realisations. Figure 1 shows our numerical results, which agree very well with the theoretical prediction. 


\section{Alternative Formulation Using Casimir Operators}

The derivation of the stationary shape distribution in Sect. 5 proceeded by setting up Langevin equations for the evolution of the variables $z$ and $\phi$ (which label positions on the Kendall sphere of triangle shapes). This Langevin process was used to obtain a onedimensional Fokker-Planck equation for $z$, after first concluding that the probability density is uniform in $\phi$. An alternative approach is to derive a Fokker-Planck equation for the distribution of all of the variables $z, \phi, \theta$ and $R$ which parametrise the relative disposition of three points in the plane, and to solve the resulting four-dimensional Fokker-Planck equation by separation of variables. This alternative method makes plain the invariance properties under the group of rotation, $S O(2)$ and the group of dilations, and in the case of the random strain model, under the group of unitary matrices, $S L(2)$ [12], at the cost of complicated algebraic manipulations, which we do not present in full detail here. These invariances are expressed via the introduction of the Casimir operators, $\hat{\mathbf{L}}^{2}, \hat{\mathbf{G}}^{2}$ and $\hat{\Lambda}$ corresponding respectively to $S O(2), S L(2)$ and the group of dilations [12]. Consistent with the convention introduced in [12], we use for the purposes of this section the coordinate $\zeta=\operatorname{det}(\mathbf{w})$ to characterise the size of the triangle, rather than the radius of gyration $R$. In fact, triangles can be parametrized either by the set of coordinates $(z, \phi, \chi, \zeta)$ coordinates, as in [1,12], or by the set of coordinates $(z, \phi, \chi, R)$ used so far. Note that our notation differ from those of [12] and [1], where the coordinates $(z, \phi, \chi, \zeta)$ were denoted $(w, \chi, \theta, \zeta)$ (we changed the notation because it is conventional to use $(z, \phi)$ as the polar projection and azimuthal angle on a sphere).

\subsection{Fokker-Planck Equation for Random Strain Model}

Here we consider how to describe the random strain process using a Fokker-Planck equation. We parametrize a triangle by the two vectors, $\boldsymbol{u}_{a}, a=1,2$, introduced in Eq. (3). These vectors are specified by four coordinates $u_{a, i}$, and we start by describing the random strain process by a Fokker-Planck equation expressed in terms of these four variables. Under the action of the random strain process, these vectors evolve according to

$$
\boldsymbol{u}_{a}=\mathbf{a}(t) \boldsymbol{u}_{a}
$$

where $\mathbf{a}(t)$ is a random $2 \times 2$ matrix. The antisymmetric part of $\mathbf{a}(t)$ corresponds to an overall rotation. Since we are interested only in the shape, we ignore the overall rotation and dilation, and consider only traceless symmetric matrices.

By integrating equation (66) with respect to time and iterating the resulting equation, in the limit of small values of the time $\delta t$ one obtains:

$u_{a, i}(\delta t)=u_{a, i}(0)+\int_{0}^{\delta t} \mathrm{~d} t^{\prime} a_{i, j}\left(t^{\prime}\right) u_{a, j}(0)+\int_{0}^{\delta t} \mathrm{~d} t^{\prime} \int_{0}^{t^{\prime}} \mathrm{d} t^{\prime \prime} a_{i k}\left(t^{\prime}\right) a_{k, j}\left(t^{\prime \prime}\right) u_{a, j}(0)+\mathcal{O}\left(\delta t^{3 / 2}\right)$

(here and in the remainder of this section summation is implied when indices are repeated). In Eq. (67), the term written as a single integral has zero mean, and a variance growing like $\delta t$. It is therefore a diffusion term. The term written with a double integral has a mean of order $\delta t$; it corresponds to the drift term. Higher order terms are formally smaller order terms in $\delta t$. To make the connection with the formulation used in Sect. 3, the stochastic increment in Eqs. (22) and (20) may be written

$$
\delta \mathbf{A}=\int_{t}^{t+\delta t} \mathrm{~d} t^{\prime} \mathbf{a}\left(t^{\prime}\right)
$$


We assume that the coefficients of the matrix $\mathbf{a}(t)$ are $\delta$-correlated in time. Using the constraint that $\mathbf{a}$ is symmetric, traceless, and has rotationally invariant statistics, we find

$$
\left\langle a_{i j}(t) a_{k l}(0)\right\rangle=\left\langle a^{2}\right\rangle \times \delta(t) \times\left(\delta_{i k} \delta_{j l}+\delta_{i l} \delta_{j k}-\delta_{i j} \delta_{k l}\right),
$$

where $\left\langle a^{2}\right\rangle$ is the only parameter that characterizes the amplitude of the noise. This relation is equivalent to Eqs. (20) and (21), with $\left\langle a^{2}\right\rangle=2 D_{s}$. The use to the relation (67) for advancing the variables $u_{a, i}$ amounts to using the physically correct Stratonovich procedure to integrate the stochastic model (1).

From Eqs. (67) and (69) one can easily determine the Fokker-Planck equation for the random-strain process:

$$
\begin{aligned}
\partial_{t} P= & -\frac{\partial}{\partial u_{a, i}}\left(v_{a, i} P\right)+\frac{\left\langle a^{2}\right\rangle}{2} \frac{\partial^{2}}{\partial u_{a, i} \partial u_{b, k}}\left(\left[\delta_{i k} \boldsymbol{u}_{a}(0) \cdot \boldsymbol{u}_{b}(0)+u_{a, k}(0) u_{b, i}(0)\right.\right. \\
& \left.\left.-u_{a, i}(0) u_{b, k}(0)\right] P\right) \\
\equiv & -\frac{\partial}{\partial u_{a, i}}\left(v_{a, i} P\right)+\frac{\left\langle a^{2}\right\rangle}{2} \hat{L}_{d} P
\end{aligned}
$$

where $v_{a, i}$ is the drift term velocity, obtained by taking the average of (67), and $L_{d}$ is a diffusion operator containing second derivatives. For the drift term we find

$$
v_{a, i}=\left\langle a^{2}\right\rangle u_{a, i}
$$

The term representing the diffusion term in the Fokker-Planck operator has a more complex structure. We find it convenient to write $L_{d}$ as the sum of three operators,

$$
\begin{aligned}
& \hat{L}_{d}^{1} \cdot P \equiv \frac{\partial^{2}}{\partial u_{a, i} \partial u_{b, i}}\left[\boldsymbol{u}_{a}(0) \cdot \boldsymbol{u}_{b}(0) P\right], \\
& \hat{L}_{d}^{2} \cdot P \equiv \frac{\partial^{2}}{\partial u_{a, i} \partial u_{b, j}}\left[u_{a, j}(0) \cdot u_{b, i}(0) P\right], \\
& \hat{L}_{d}^{3} \cdot P \equiv \frac{\partial^{2}}{\partial u_{a, i} \partial u_{b, j}}\left[u_{a, i}(0) \cdot u_{b, j}(0) P\right] .
\end{aligned}
$$

\subsection{Representation Using Casimir Operators}

Now consider how the Fokker-Planck equation (70) may be transformed from Cartesian coordinates to the more convenient set $(z, \phi, \chi, \zeta)$ which separate the actions of shape, rotation, and scale transformations. As a consequence, we shall see how the operator may also be decomposed into Casimir operators for these fundamental group operations.

The drift term in the Fokker-Planck equation, $\partial_{a}^{i}\left(v_{a}^{i} P\right)$, makes a contribution which takes a very simple form, involving only the coordinate $\zeta$ :

$$
\frac{\partial}{\partial u_{a, i}}\left(v_{a, i} P\right)=2\left\langle a^{2}\right\rangle\left(2 \zeta \frac{\partial P}{\partial \zeta}+4 P\right)
$$

Each of the three operators in Eq. (72) can be written in terms of the Casimir operators of $S O(2)\left(\hat{\mathbf{L}}^{2}\right)$, of $S L(2)\left(\hat{\mathbf{G}}^{2}\right)$ and of the dilation operator, $\hat{\Lambda}$. 
The angular momentum operators are, up to a factor $\pm \mathrm{i}$,

$$
\hat{L}^{i j} \cdot P=\left(u_{a, i} \frac{\partial}{\partial u_{a, j}}-u_{a, j} \frac{\partial}{\partial u_{a, i}}\right) P
$$

and in this two-dimensional problem the corresponding Casimir operator is

$$
\hat{\mathbf{L}}^{2}=\frac{1}{2} \hat{L}^{i j} \hat{L}^{j i}
$$

A straightforward calculation leads to:

$$
\hat{\mathbf{L}}^{2} P=\frac{\partial^{2}}{\partial u_{a, i} \partial u_{b, j}}\left(u_{a, j} u_{b, i} P\right)-\frac{\partial^{2}}{\partial u_{a, i} \partial u_{b, i}}\left(\boldsymbol{u}_{a} \cdot \boldsymbol{u}_{b} P\right)-(d-1)\left(d^{2}+\hat{\Lambda}\right) P
$$

where $d=2$ is the space dimension, and where

$$
\hat{\Lambda}=u_{a, i} \frac{\partial}{\partial u_{a, i}} .
$$

The operators that generate the representations of $S L(2)$ are:

$$
\hat{G}^{a b}=u_{a, i} \frac{\partial}{\partial u_{b, i}}-\frac{\delta_{a b}}{2} \hat{\Lambda} .
$$

The operator $G^{a b}$ and $\Lambda$ commute with each other.

The operator $\mathbf{G}^{2} \equiv G^{a b} G^{b a}$ can be easily expressed in the form:

$$
\hat{\mathbf{G}}^{2}=\frac{\partial^{2}}{\partial u_{b, i} \partial u_{a, j}}\left(u_{a, i} u_{b, j} \cdot\right)-\frac{1}{d}\left(\hat{\Lambda}+d^{2}\right)\left(\hat{\Lambda}+2 d^{2}\right) .
$$

Last, we express $\hat{\Lambda}^{2}$ :

$$
\hat{\Lambda}^{2}=\frac{\partial^{2}}{\partial u_{a, i} \partial u_{b, j}}\left(u_{a, i} u_{b, j} \cdot\right)-\left(2 d^{2}+1\right)\left(d^{2}+\hat{\Lambda}\right)+d^{4} .
$$

Thus, the 3 operators $\hat{L}_{d}^{i}$ are:

$$
\begin{aligned}
\hat{L}_{d}^{1} & =-\hat{\mathbf{L}}^{2}+\hat{\mathbf{G}}^{2}+\frac{1}{d}\left(\hat{\Lambda}+d^{2}\right)\left(\hat{\Lambda}+2 d^{2}\right)-(d-1)\left(\hat{\Lambda}+d^{2}\right), \\
L_{d}^{2} & =\hat{\mathbf{G}}^{2}+\frac{1}{d}\left(\hat{\Lambda}+d^{2}\right)\left(\hat{\Lambda}+2 d^{2}\right), \\
L_{d}^{3} & =\hat{\Lambda}^{2}+\left(2 d^{2}+1\right)\left(\hat{\Lambda}+d^{2}\right)-d^{4} .
\end{aligned}
$$

With these expressions, one can simply obtain the expression for $\hat{L}_{d}$ :

$$
\hat{L}_{d} \cdot U=\left(-\hat{\mathbf{L}}^{2}+2 \hat{\mathbf{G}}^{2}+2 \hat{\Lambda}+8\right) U
$$

The terms $\hat{\mathbf{L}}^{2}, \hat{\mathbf{G}}^{2}$ and $\hat{\Lambda}^{2}$ all have a simple expression in terms of the variables $(z, \phi, \chi, \zeta)[12]$. Specifically,

$$
\hat{\mathbf{L}}^{2}=\frac{\partial^{2}}{\partial \chi^{2}},
$$




$$
\begin{aligned}
& \hat{\mathbf{G}}^{2}=2 z^{2} \frac{\partial}{\partial z}\left[\left(1-z^{2}\right) \frac{\partial}{\partial z}\right]+\frac{z^{2}}{2\left(1-z^{2}\right)}\left(\frac{\partial^{2}}{\partial \chi^{2}}+\frac{\partial^{2}}{\partial \phi^{2}}-2 z \frac{\partial^{2}}{\partial \chi \partial \phi}\right), \\
& \hat{\Lambda}^{2}=4\left(\zeta^{2} \frac{\partial^{2}}{\partial \zeta^{2}}+\zeta \frac{\partial}{\partial \zeta}\right) .
\end{aligned}
$$

Thus, the operator $\hat{L}_{d}$ reads:

$$
\begin{aligned}
\hat{L}_{d} \cdot U= & 4 z^{2} \frac{\partial}{\partial z}\left[\left(1-z^{2}\right) \frac{\partial U}{\partial z}\right]+\frac{z^{2}}{\left(1-z^{2}\right)}\left(\frac{\partial^{2}}{\partial \phi^{2}}-2 z \frac{\partial^{2}}{\partial \chi \partial \phi}\right) U \\
& +\frac{1}{1-z^{2}} \frac{\partial^{2} U}{\partial \phi^{2}}+4 \zeta \frac{\partial U}{\partial \zeta}+8 U .
\end{aligned}
$$

\subsection{Relation to Formulation in Sect. 3}

Equation (90) enables us to express the Fokker-Planck operator in terms of the coordinates $(z, \phi, \chi, \zeta)$. Let us consider how to obtain the results developed in Sect. 3 using this representation of the Fokker-Planck operator. Because we intend to look at solutions which are independent of the rotation angle $\chi$, the diffusion part of the operator is:

$$
L_{d} \cdot U=4 z^{2} \frac{\partial}{\partial z}\left[\left(1-z^{2}\right) \frac{\partial U}{\partial z}\right]+\frac{z^{2}}{\left(1-z^{2}\right)} \frac{\partial^{2} U}{\partial \phi^{2}}+4 \zeta \frac{\partial U}{\partial \zeta}+8 U .
$$

Thus, the full Fokker-Planck equation, expressed as in Eq. (70), is simply

$$
\partial_{t} P=\left\langle a^{2}\right\rangle\left(2 z^{2} \frac{\partial}{\partial z}\left[\left(1-z^{2}\right) \frac{\partial P}{\partial z}\right]+\frac{z^{2}}{2\left(1-z^{2}\right)} \frac{\partial^{2} P}{\partial \phi^{2}}\right) .
$$

As expected, this is independent of $\zeta$. However, its structure appears hard to reconcile with the results for the random strain model in Sect. 3. In order to make the connections clear, we first remark that this is an equation for a probability density $P$ expressed as a function of the four original variables, $u_{a, i}, a=1,2, i=1,2$. The transformation to the new variables $\zeta, \chi, z, \phi$ involves the Jacobian

$$
\frac{D\left(u_{1,1}, u_{1,2}, u_{2,1}, u_{2,2}\right)}{D(z, \phi, \chi, \zeta)}=\frac{\zeta}{z^{2}}
$$

The total probability is obtained by integrating $P$ over all the $u_{a, i}$. After transforming to the $(z, \phi, \chi, \zeta)$ variables it is more convenient to deal with a probability density $P^{\prime}(z, \phi, \chi, \zeta)$ which is a conserved quantity in these variables. In the $(z, \phi, \chi, \zeta)$ variables, the normalization condition reads:

$$
\begin{aligned}
& \int \mathrm{d} u_{1,1} \mathrm{~d} u_{1,2} \mathrm{~d} u_{2,1} \mathrm{~d} u_{2,2} P\left(u_{1,1}, u_{1,2}, u_{2,1}, u_{2,2}\right) \\
& =\int \mathrm{d} \zeta \mathrm{d} \chi \mathrm{d} z \mathrm{~d} \phi P(\zeta, \chi, z, \phi) \frac{\zeta}{z^{2}}=\int \mathrm{d} \zeta \mathrm{d} \chi \mathrm{d} z \mathrm{~d} \phi P^{\prime}(\zeta, \chi, z, \phi)
\end{aligned}
$$

If we rewrite Eq. (92) in terms of $P^{\prime}$ we obtain

$$
\partial_{t} P^{\prime}=\left\langle a^{2}\right\rangle\left(2 \frac{\partial}{\partial z}\left[\left(1-z^{2}\right) \frac{\partial z^{2} P^{\prime}}{\partial z}\right]+\frac{z^{2}}{2\left(1-z^{2}\right)} \frac{\partial^{2} P^{\prime}}{\partial \phi^{2}}\right) .
$$


Using some elementary manipulation of the part in $z$ of the evolution operator, one can rewrite:

$$
\frac{\partial}{\partial z}\left[\left(1-z^{2}\right) \frac{\partial z^{2} P^{\prime}}{\partial z}\right]=\frac{\partial^{2}}{\partial z^{2}}\left[z^{2}\left(1-z^{2}\right) P^{\prime}\right]-\frac{\partial}{\partial z}\left(-2 z^{3} P\right)
$$

which agrees with the conclusion that the change in shape can be described by a diffusion $z^{2}\left(1-z^{2}\right)$ and a drift $-2 z^{3}$.

\subsection{Fokker-Planck Equation for Brownian Process}

Next consider the Fokker-Planck equation for the relative Brownian diffusion of three points, and its relation to the derivation of Kendall's result on diffusion which as discussed in Sect. 4. After a lengthy calculation, this is found to take the following form

$$
\begin{aligned}
\partial_{t} P= & \frac{D_{\mathrm{b}}}{z \zeta}\left[2 \zeta^{2} \frac{\partial^{2} P}{\partial \zeta^{2}}+2 z^{2} \partial_{z}\left[\left(1-z^{2}\right) \partial_{z} P\right]\right. \\
& +\frac{z^{2}}{2\left(1-z^{2}\right)}\left(\frac{\partial^{2} P}{\partial \phi^{2}}+\frac{\partial^{2} P}{\partial \chi^{2}}-2 z \frac{\partial^{2} P}{\partial \chi \partial \phi}\right) \\
& \left.+4\left(1-z^{2}\right) z \zeta \frac{\partial^{2} P}{\partial z \partial \zeta}\right]
\end{aligned}
$$

where $D_{\mathrm{b}}$ is the amplitude of the additive noise term:

$$
\partial_{t} u_{a, i}=v_{a, i} \quad \text { with }\left\langle v_{a, i}(t) v_{b, j}(0)\right\rangle=2 D_{\mathrm{b}} \delta(t) \delta_{a b} \delta_{i j} .
$$

Because we chose to consider a process that does not change the radius of the solution. This suggests that the evolution will be simple in the variables $(z, \phi, \chi, R)$. Indeed, the evolution operator in these variables is simply:

$$
\partial_{t} P=D_{\mathrm{b}} \times\left(\frac{\partial}{\partial z}\left[\left(1-z^{2}\right) \frac{\partial}{\partial z} P\right]+\frac{1}{1-z^{2}}\left(\partial_{\phi^{2}}+\partial_{\chi^{2}}-2 z \partial_{\phi} \partial_{\chi}\right) P\right)
$$

The Jacobian of the transformation to the variables $(z, \phi, \chi, R)$ is proportional to $R^{3}$, i.e., it does not depend on the angular variables. The simple identity:

$$
\frac{\partial}{\partial z}\left[\left(1-z^{2}\right) \frac{\partial P}{\partial z}\right]=\frac{\partial^{2}}{\partial z^{2}}\left[\left(1-z^{2}\right) P\right]-\frac{\partial}{\partial z}(-2 z P)
$$

immediately leads to the conclusion that the evolution of $z$ results from a diffusion (coefficient $\propto\left(1-z^{2}\right)$ ) and a drift (coefficient $\propto-2 z$ ), as obtained in Sect. 4.

\section{Summary}

We have analysed the simplest model which could describe the shape statistics for triplets of points advected in a two-dimensional random flow. The model combines Brownian processes which describe a random strain with a Brownian motion of the triangle vertices. We characterised the shape using Kendall's spherical representation of the manifold of triangle shapes. The steady-state distribution is uniform in the azimuthal coordinate $\phi$ but has a Lorentzian form in the polar altitude $z$, depending upon a single dimensionless parameter. 
The model considered in this work can be viewed as a simplification of the models proposed in $[1,7]$, which are taking into account the hierarchy of time scales in a turbulent flow. Understanding the shape distributions predicted by these models will require some generalization of the ideas developed in this work. Still, we expect that the solution discussed here provides a starting point for future analysis of the shape distribution of triangles, or more complicated sets of points transported by turbulent flows.

Acknowledgements We acknowledge R. Guichardaz for his comments on our manuscript. A.P. has been supported by the grant from A.N.R. "TEC 2". We acknowledge the support of the European COST Action MP0806.

\section{References}

1. Castiglione, P., Pumir, A.: Evolution of triangles in a two-dimensional turbulent flow. Phys. Rev. E 64, 056303 (2001)

2. Cressman, J.R., Davoudi, J., Goldburg, W.I., Schumacher, J.: Eulerian and Lagrangian studies in surface flow turbulence. New J. Phys. 6, 53 (2004)

3. de Chaumont Quitry, A., Kelley, D.H., Ouellette, N.T.: Mechanisms driving shape distortion in twodimensional flow. Europhys. Lett. 94, 64006 (2011)

4. Kendall, D.G.: The diffusion of shape. Adv. Appl. Probab. 9, 428-430 (1977)

5. Kendall, D.G.: Shape manifolds, procrustean metrics, and complex projective spaces. Bull. Lond. Math. Soc. 16, 81-121 (1984)

6. Kendall, W.S.: A diffusion model for Bookstein triangle shape. Adv. Appl. Probab. 30, 317-334 (1998)

7. Pumir, A., Shraiman, B., Chertkov, M.: Geometry of Lagrangian dispersion in turbulence. Phys. Rev. Lett. 85, 5324 (2000)

8. Biferale, L., Boffetta, G., Celani, A., Devenish, B.J., Lanotte, A., Toschi, F.: Multiparticle dispersion in fully developed turbulence. Phys. Fluids 17, 111701 (2005)

9. Xu, H., Ouellette, N.T., Bodenschatz, E.: Evolution of geometric structures in intense turbulence. New J. Phys. 10, 013012 (2008)

10. Hackl, J.F., Yeung, P.K., Sawford, B.L.: Multi-particle and tetrad statistics in numerical simulations of turbulent relative dispersion. Phys. Fluids 23, 065103 (2011)

11. Mydlarski, L., Pumir, A., Shraiman, B.I., Siggia, E.D., Warhaft, Z.: Structure and multi-point correlators for turbulent advection. Phys. Rev. Lett. 81, 4373-4376 (1998)

12. Shraiman, B.I., Siggia, E.D.: Anomalous scaling for a passive scalar near the Batchelor limit. Phys. Rev. E 57, 2965-2977 (1998)

13. Shraiman, B.I., Siggia, E.D.: Scalar turbulence. Nature 405, 639-646 (2000)

14. Falkovich, G., Gawedzki, K., Vergassola, M.: Particles and fields in turbulence. Rev. Mod. Phys. 73, 913-975 (2000)

15. Gat, O., Zeitak, R.: Multiscaling in passive scalar advection as stochastic shape dynamics. Phys. Rev. E 57, 5511-5519 (1998)

16. Balkovisky, E., Chertkov, M., Kolokolov, I., Lebedev, V.: Fourth-order correlation function of randomly advected passive scalar. JETP Lett. 61, 1049 (1995)

17. Pumir, A., Shraiman, B., Siggia, E.D.: Perturbation theory for $\delta$-correlated model of passive scalar advection near the Batchelor limit. Phys. Rev. E 55, R1263-1266 (1997)

18. Chertkov, M., Kolokolov, I., Lebedev, V.: Strong effect of weak diffusion on scalar turbulence at large scales. Phys. Fluids 19, 101703 (2007)

19. van Kampen, N.G.: Stochastic Processes in Physics and Chemistry, 2nd edn. North-Holland, Amsterdam (1981) 